\title{
The Effectiveness of Self-Management Mobile Health Technology in Chronic Disease: A Systematic Review
}

\author{
N Montaseri ${ }^{1 ;}$; Langarizadeh ${ }^{2,}$; A Z Bayani ${ }^{3} ;$ F Moghbeli $^{2}$ \\ MSc, School of Allied Medical Sciences, Tehran University of Medical Sciences, Tehran, Iran \\ ${ }_{2}^{2}$ Ph.D, School of Health Management and Information Science, Iran University of Medical Sciences, Tehran, Iran \\ ${ }^{3}$ MSc, Health Information Management Department, School of Allied Medical Sciences, Tehran University of Medical Sciences, Tehran, Iran \\ ${ }^{*}$ Corresponding author: M Langarizadeh, Health Management and Information Department School of Science, Hemmat Highway, Tehran, Iran. Tel: +98-9198616016, \\ E-mail: langarizadeh.m@iums.ac.ir
}

Received: 11 Dec 2016

Accepted: 01 Jan 2017

Epub: 23 Feb 2017

Ppub: 15 Jan 2018

\begin{abstract}
Background: Chronic diseases have a high prevalence in the world and an intense impact on the life of patients. For enhancing the health care in chronic diseases and reducing costs, the self-management approaches using technology are helpful in improving patient care and better endurance of coarseness of the diseases. Due to the high use of mobile in societies, the usage of this kind of technology, for better management of chronic diseases and the impacts of it, are studied in various studies.

Objectives: We conducted a systematic review to investigate the efficiency and effectiveness of m-health technology in self-management of chronic diseases.

Methods: We searched MEDLINE/PubMed electronically for articles published between 01/01/2006 and 08/18/2016.The inclusion criteria were set based on the type of study and beneficial or harmful effects of mobile technology based intervention in relation to chronic disease and self-management. A total of 78 articles were identified based on the searches conducted in MEDLINE from PubMed. After excluding duplicates $(\mathrm{n}=34)$, we screened titles and abstracts of 44 articles based on the inclusion criteria. The remaining articles matched with the inclusion criteria $(n=38)$ were reviewed in full text, and 15 articles were excluded based on the exclusion criteria. Finally, 23 articles complied with our eligibility criteria and were included in this study.

Results: Of the 23 studies we reviewed, 13 studies assessed the impact of using mobile technology in the diseases and all of them reported a good or high level of benefits as well as improved clinical outcomes. A total of 7 studies had the purpose of investigating usability, however, only 3 of them who concluded the present mobile technology qualified as a high level of usability. Six studies explored the feasibility of the mobile technology and all studies who reported mobile technology were feasible. Three studies explored the acceptability of the mobile technology and all studies reported a high level of acceptability. A total of 4 articles had the purpose of investigating satisfaction and all of them demonstrated high satisfaction of mobile technology.

Conclusions: The evidence indicates the potential of m-health technology in improving symptom management through selfmanagement interventions.

Keywords: M-Health; Self-Management; Chronic Diseases
\end{abstract}

